Draft version November 17, 2020

Preprint typeset using $\mathrm{IAT}_{\mathrm{E}} \mathrm{X}$ style emulateapj v. 12/16/11

\title{
PHENOMENOLOGICAL GRAVITATIONAL PHASE TRANSITION: RECONCILIATION BETWEEN THE LATE AND EARLY UNIVERSE
}

\author{
MARZIEH FARHANG AND Nima KhosRAVI \\ Department of Physics, Shahid Beheshti University, 1983969411, Tehran Iran \\ Draft version November 17, 2020
}

\begin{abstract}
In this work we investigate whether a certain phenomenological extension to the general relativity (GR), in the form of a gravitational phase transition (GPT) in the Universe, can reduce the external Planck tensions with the local Hubble measurements and the distribution of matter, characterized by $\sigma_{8}$, as well as its internal inconsistencies in the lensing amplitude and the low-high $\ell$ parameter estimates. We introduce new degrees of freedom into the background and the two scalar perturbation equations in the Newtonian gauge, with simultaneous transitions from an early gravitational phase equivalent to GR toward a late phase. We model the transition as a "tanh" parametrized by the transition redshift $z_{\mathrm{t}}$ and width $\alpha$, with amplitudes $\Lambda(z)$ and $(\mu(z), \gamma(z))$ for the background and perturbations respectively. We verify the consistency of the datasets used in this work in the GPT framework and confirm that the individual tensions do not require conflicting transitions. We find that the joint datasets prefer a recent transition at $z_{\mathrm{t}} \approx 0.9$ in the background and perturbed Einstein equations, driven mainly by the local Hubble measurement. This transition relaxes all the tensions considered in this work.
\end{abstract}

\section{INTRODUCTION AND MOTIVATIONS}

The standard model of cosmology, aka $\Lambda$ CDM, got well established during the golden era of cosmology, thanks to the high precision data, in particular the fluctuations in the temperature and polarization of the cosmic microwave background radiation (CMB) (Planck Collaboration et al. 2020a). Recently, however, several recent independent observations have put the vanilla $\Lambda \mathrm{CDM}$ model under tension. The most significant is the $H_{0}$ tension which emerged with the precise local measurements of the Hubble constant, $H_{0}=74.03 \pm 1.42 \mathrm{~km} / \mathrm{s} / \mathrm{Mpc}$ (Riess et al. 2019, 2018, 2016). This value is in more than $4 \sigma$ tension with the $\Lambda \mathrm{CDM}$ prediction of Planck, $H_{0}=67.36 \pm 0.54$ (Planck Collaboration et al. 2020a). The tension is supported by other independent local measurements (Wong et al. 2020; Freedman et al. 2019; Huang et al. 2019; Khetan et al. 2020; Pesce et al. 2020). A milder $2 \sigma-3 \sigma$ tension is also reported between late measurements of the current matter distribution, as inferred from Planck, $S_{8}=\sigma_{8} \sqrt{\Omega_{m} / 0.3}=0.834 \pm 0.016$, and the measurements by DES $S_{8}=0.792 \pm 0.024$ (Abbott et al. 2018) and by KiDS $S_{8}=0.766_{-0.014}^{+0.020}$ from weak gravitational lensing and redshift space distortion (Heymans et al. 2020).

Besides these tensions, there are also reports on internal discrepancies in the Planck temperature dataset when interpreted in the $\Lambda \mathrm{CDM}$ framework (Planck Collaboration et al. 2020a). The first is the $\sim 2 \sigma$ deviation of the lensing amplitude, $A_{\text {lens }}$, from unity which is required by the theoretical consistency of the theory, and is also consistent with the $A_{\text {lens }}$ estimation from the Planck four point correlation function. The second is the inconsistency of the measurements of certain cosmological parameters, derived independently from the low and high CMB multipoles ${ }^{1}$. To make this list of CMB-based cos-

m_farhang@sbu.ac.ir; n-khosravi@sbu.ac.ir

1 This inconsistency may be related to the former anomaly in mic curiosities complete, we also mention that there are also the broadly addressed CMB spatial anomalies which challenge the isotropy and homogeneity of the Universe such as the dipole modulation, the quadrupole-octopole alignment and the CMB cold spot (Planck Collaboration et al. 2020b) .

There have been extensive studies of all these temporal and spatial tensions and anomalies, in particular, of the possibility of them being produced by yet unexplained systematics. Moreover, many recent beyond $-\Lambda \mathrm{CDM}$ proposals have emerged by relaxing some of its assumptions. These include treating the neutrino masses as free parameters, however, found to increase the $\sigma_{8}$ tension (Planck Collaboration et al. 2020a), tweaking the physics of the early Universe (Keeley et al. 2020), and to look for windows in the dark side of the Universe, in both dark matter (see, e.g., Vattis et al. 2019)) and dark energy sectors, (see, e.g., Keeley et al. 2019; Di Valentino et al. 2020c; Zhao et al. 2017; Khosravi et al. 2019; Yan et al. 2020; Braglia et al. 2020; Raveri 2020; Poulin et al. 2019; Di Valentino et al. 2017; Gómez-Valent et al. 2020; Lucca \& Hooper 2020; Yang et al. 2018b,a; Di Valentino et al. 2020a,b; Li \& Shafieloo 2019). The physics of critical phenomena is also a recent proposal to support the physical motivation for a phase transition in the behavior of dark energy and is phenomenologically explored at the background level (Banihashemi et al. 2019, 2020).

In this paper we investigate whether a gravitational phase transition, referred to GPT, can address the above internal and external tensions. For this purpose, we explore the gravitational parameter space from an effective viewpoint. Einstein's intuition in general relativity (GR) was absolutely genius. Yet, a more systematic approach to the theory of gravitation is the formalism of the effective field theory (EFT) which includes all theoretically possible terms, e.g., all those satisfying a required sym-

the lensing amplitude (Planck Collaboration et al. 2020a). 
metry (Gubitosi et al. 2013; Bloomfield et al. 2013). In this framework GR can be considered as a special case, obtained through the fine-tuning of some of the EFT parameters. GR is also the most economic formulation in terms of the minimum number of degrees of freedom (dof's) required for a gravitational theory. Any modification to it will therefore propagate new dof's. In the EFT approach, the modification to the background gravity can be modeled through replacing the cosmological constant by a time-dependent field ${ }^{2}$, while two new scalar dof's are required to describe modification to perturbation equations in the linear level in the Newtonian gauge.

In this work we parametrize these dof's by the three functions ${ }^{3} \Lambda(z)$ and $(\mu(z), \Sigma(z))$, assumed to be constant in $\Lambda$ CDM with $\Lambda(z)=\Lambda$ and $(\mu(z), \Sigma(z))=(1,-1)$. Here we parametrize their redshift dependence by a "tanh". For this assumption, we are motivated by the idea of a possible phase transition in the dark energy, proposed to relax the cosmological tensions ${ }^{4}$. We use "tanh" to phenomenologically model this phase transition in the mean field approximation. We explore the space spanned by the new GPT dof's to investigate the consistency of the various cosmological datasets in this framework and to investigate the power of this proposal in relaxing the tensions.

In the rest of this paper we first introduce the phenomenological effective framework of GPT (Section 2) and the datasets (Section 3). The results on the resolution of the external and internal tensions are presented in Sections 4 and 5 and we conclude in Section 6.

\section{THE GPT MODEL}

In this work we propose a single late-time transition in the behavior of the gravitational theory, modeled phenomenologically by a "tanh" function. By late time we refer to any epoch between the last scattering surface and now. We assume the early-time gravity, well before the transition, is described by GR. This assumption could be relaxed in principle in the cost of increasing the number of free parameters of the theory.

We assume our Universe is isotropic, homogeneous and flat, with the background evolution described by

$$
H^{2}(z)=H_{0}^{2}\left[\Omega_{r}(1+z)^{4}+\Omega_{m}(1+z)^{3}+\Omega_{\Lambda}(z)\right]
$$

and with linear scalar perturbations described by

$$
d s^{2}=a^{2}(\tau)\left[-(1+2 \Psi) d \tau^{2}+(1-2 \Phi) d r^{2}\right]
$$

in the Newtonian gauge. Here $a(\tau)$ is the scale factor and $\Psi$ and $\Phi$ characterize the scalar perturbations. Phenomenologically, the modified Einstein equations in Fourier space describing the evolution of perturbations

\footnotetext{
2 The Newton constant $G_{\mathrm{N}}$ is also a possibility. For our purpose, however, it is degenerate with the other parameters.

3 Note that in principle these variables can be scale dependent as $\mu(k, z)$ and $\gamma(k, z)$.

4 In this work we do not address the spatial CMB anomalies. However, we showed in Banihashemi et al. (2019) that a phase transition inspired by the physics of critical phenomena can provide a rich framework for simultaneous exploration of spatial anomalies and temporal tensions.
}

in the linear level are given by

$$
\begin{aligned}
& k^{2} \Psi=-\mu(z) 4 \pi G a^{2}[\rho \Delta+3(\rho+P) \sigma] \\
& k^{2}[\Phi-\gamma(z) \Psi]=\mu(z) 12 \pi G a^{2}(\rho+P) \sigma
\end{aligned}
$$

where $\mu(z)$ and $\gamma(z)$ encode all the information about the departure of the gravitational theory from Einstein gravity. Alternatively, Equation (4) can be replaced by

$$
k^{2}(\Phi+\Psi)=8 \pi G a^{2} \Sigma(z) \rho \Delta
$$

which is particularly useful for characterizing the gravitational lensing.

We model the modification to the background by a transition in the cosmological constant

$$
\Omega_{\Lambda}(z)=\Omega_{\Lambda}^{\text {early }}+\Delta_{\Lambda} \frac{1+\tanh \left[\alpha\left(z_{\mathrm{t}}-z\right)\right]}{2} .
$$

where $z_{\mathrm{t}}$ is the transition redshift and $\alpha$ is the inverse of transition's width. Flatness requires $\Omega_{\mathrm{r}}+\Omega_{\mathrm{m}}+\Omega_{\Lambda}=1$, where $\Omega_{\Lambda} \equiv \Omega_{\Lambda}(z=0)=\Omega^{\text {early }}+\Delta_{\Lambda}\left(1+\tanh \left(\alpha z_{\mathrm{t}}\right)\right) / 2$. Similarly, the modifications to the perturbation equations are characterized by transitions in $\mu(z)$ and $\gamma(z)$,

$$
\begin{aligned}
& \mu(z)=\mu^{\text {early }}+\Delta_{\mu} \frac{1+\tanh \left[\alpha\left(z_{\mathrm{t}}-z\right)\right]}{2}, \\
& \gamma(z)=\gamma^{\text {early }}+\Delta_{\gamma} \frac{1+\tanh \left[\alpha\left(z_{\mathrm{t}}-z\right)\right]}{2} .
\end{aligned}
$$

where $\mu^{\text {early }}=\gamma^{\text {early }}=1$ and $\Sigma^{\text {early }}=-1$.

\section{ANALYSIS AND DATASETS}

In this work we use the CMB temperature and $E$-mode polarization power spectra as measured by Planck 2018 data, the same as the reference likelihood in the Planck Collaboration et al. (2020a). We refer to it as Pl18. In the final analysis of this paper in section 6 , where the results for the joint datasets are presented, we also include the Planck CMB lensing, labeled as + lensing.

As the primary late time data point we use the most recent Hubble constant measurement from Riess et al. (2019), given by $H_{0}=74.03 \pm 1.42 \mathrm{~km} / \mathrm{s} / \mathrm{Mpc}$, referred to as R19 throughout the paper. We also use the cosmic shear and galaxy clustering measurements, as well as their cross correlation, from the first year observation of the Dark Energy Survey (Abbott et al. 2018), referred to as DES in the following sections. In some cases we assume a futuristic observational scenario for $H_{0}$ measurement, referred to as H073, where the current best-fit value of $H_{0}$ is retained with much tighter bounds.

The parameter space in this work consists of the standard cosmological parameters, $\left(\Omega_{\mathrm{b}} h^{2}, \Omega_{\mathrm{c}} h^{2}, \theta, A_{\mathrm{s}}, n_{\mathrm{s}}, \tau\right)$, and the parameters effectively describing the GPT, $\left(z_{\mathrm{t}}, \alpha, \Delta_{\Lambda}, \Delta_{\mu}, \Delta_{\gamma}\right)$ (see Equations 6 and 7 ). In section $5.2, A_{\text {lens }}$ is also set free as part of the study of the internal consistency of the Planck temperature dataset in the GPT framework. We use the publicly available Markov-Chain Monte-Carlo (MCMC) code, CosmoMC ${ }^{5}$, to sample the parameter space.

\section{RESULTS I: $H_{0}$ AND $\sigma_{8}$ TENSIONS}

\footnotetext{
5 https://cosmologist.info/cosmomc/
} 


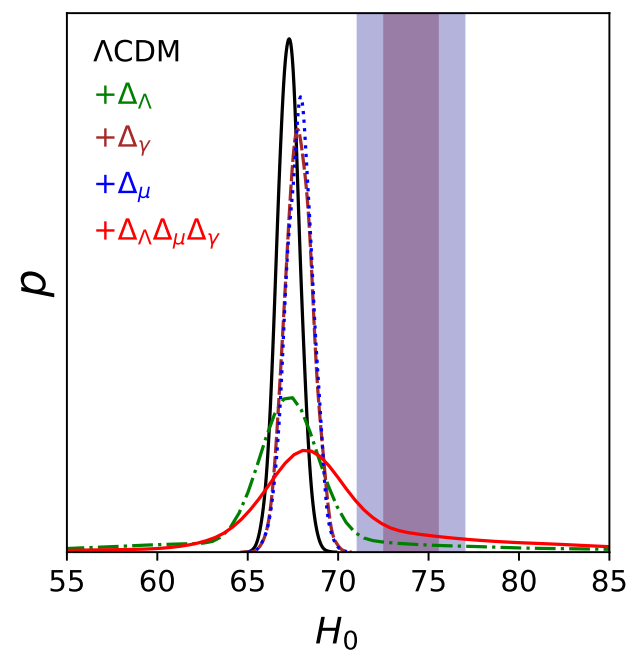

FIG. 1. - The 1D marginalized $H_{0}$ likelihood as measured by Pl18 compared for the $\Lambda$ CDM model and the GPT minimal $\left(+\Delta_{\Lambda}\right.$ $+\Delta_{\mu}$ and $\left.+\Delta_{\gamma}\right)$ and full $\left(+\Delta_{\Lambda} \Delta_{\mu} \Delta_{\gamma}\right)$ scenarios. The tension with R19 measurements (shaded purple $1-2 \sigma$ region) is relaxed with transitions in the background and perturbation dof's $\left(+\Delta_{\Lambda} \Delta_{\mu} \Delta_{\gamma}\right)$.

In this section we explore the potential of the GPT parameter space for peaceful explanation of the tensions between CMB-based inferences and R19 measurements of $H_{0}$ (Section 4.1) and DES implications for the growth of structures (Section 4.2).

\subsection{Hubble tension}

Figure 1 compares the Pl18 1D marginalized likelihoods for $H_{0}$ in the the $\Lambda \mathrm{CDM}$ and GPT scenarios. The minimal GPT scenarios, with a single GPT amplitude allowed to vary, correspond to background-only $\left(+\Delta_{\Lambda}\right)$ and perturbation-only transitions $\left(+\Delta_{\gamma}\right.$ and $\left.+\Delta_{\mu}\right)$. The full model, labeled as $+\Delta_{\Lambda} \Delta_{\gamma} \Delta_{\mu}$, includes simultaneous transitions in the background and the two perturbed Einstein equations. The shaded vertical bars indicate the $1 \sigma$ and $2 \sigma$ confidence regions of $H_{0}$ by R19. We see that the background transition opens up the Pl18 constraint on $H_{0}$ and reduces the tension. Transition in a single perturbation equation, on the other hand, by either $\Delta_{\mu}$ or $\Delta_{\gamma}$, slightly shifts the position of the $H_{0}$ likelihood peak without significant impact on the distribution width. Allowing for simultaneous transitions in the background and perturbations leads to considerable increase in the the bounds on $H_{0}$ and removes the tension.

This relaxation in the full GPT parameter space is not unexpected and can be explained as follows. The tight observational constraint on the angular size of the sound horizon, $\theta_{\mathrm{s}}=r_{\mathrm{s}} / D_{\mathrm{LSS}}$, where $r_{\mathrm{s}}=\int_{z_{\mathrm{drag}}}^{\infty} c_{\mathrm{s}} d z / H(z)$ and $D_{\mathrm{LSS}}=\int_{0}^{z_{\mathrm{LSS}}} d z / H(z)$, leads in turn to tight constraints on $\Omega_{m} h^{3}$ in $\Lambda$ CDM (Percival et al. 2002; Planck Collaboration et al. 2020a). In GPT, with a different expansion history, the degeneracy between the parameters introduced by the new dof's in $\Omega_{\Lambda}$, would lead to enhanced bounds on $\Omega_{m}$ and $H_{0}$, after marginalization over other parameters. On the other hand, there are tight constraints on matter density, $\Omega_{m} h^{2}$, due to its relatively distinct imprints on temperature power spectrum, through affecting the epoch of equality, the depth of potential wells (and thus the monopole at the last scattering) and

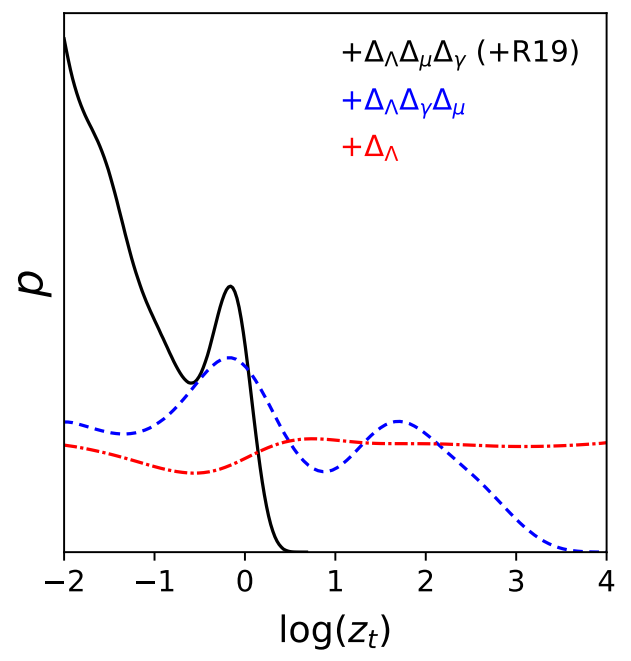

Fig. 2. - The marginalized likelihood of $\log z_{\mathrm{t}}$ as measured by Pl18 for the background and full GPT parameters. The black curve represents the likelihood by the joint Pl18+R19 for the full GPT, and prefers a transition at $z_{\mathrm{t}} \approx 0.9$. The $z_{\mathrm{t}} \approx 0$ transition is not physically motivated and therefore not further considered here.

the lensing of CMB photons. Therefore, the backgroundonly transition by itself is inadequate to explain the observed $H_{0}=73.4 \mathrm{~km} / \mathrm{s} / \mathrm{Mpc}$, and would benefit from the dof's in perturbation equations which are partially degenerate with matter density. This in turn would induce correlations with other standard parameters through the early-time imprints of matter density, which cannot be compensated by the late-time imprints of GPT dof's.

Figure 2 illustrates the distribution of the transition redshift as measured by the Pl18 and Pl18+R19 for the background and full GPT scenarios. The combined dataset prefers a very recent transition at $z \approx 0$ and also allows for a transition at $z_{\mathrm{t}} \approx 0.9$. The $z \approx 0$ scenario, imposed by the $H_{0}$ prior from R19, is the trivial reconciliation of the late $H_{0}$ with the CMB inference of $H_{0}$. Its corresponding background history respects $\Lambda \mathrm{CDM}$ until very recently, and make a sudden switch to yield the high $H_{0}$ of R19. This fine-tuned scenario is however the least physically-motivated. We therefore regard the $z_{\mathrm{t}} \approx 0.9$ as the main scenario suggested by data.

The sharp decline in the high- $z$ tail of this transition can be explained as follows. The background history is equivalent to $\Lambda \mathrm{CDM}$ at high $z$, and the accompanying transitions in $\mu$ and $\gamma$, with effectively no modification to the background, cannot solve the tension (Figure 1). This also explains the non-vanishing probability of $z_{\mathrm{t}}$ at higher redshifts for the CMB-only measurement in the background GPT scenario.

\section{2. $\sigma_{8}$ tension}

The claims of moderate tension between the Pl18 $\Lambda$ CDM-based inferences and DES probes are mostly apparent in combinations of background matter density and its fluctuations (see, e.g., Fig. 20 of Planck Collaboration et al. 2020a). We include the GPT parameters in the analysis and find the tension is relieved by the boosted parameter ranges allowed by the data,

$$
S_{8}=0.836 \pm 0.042, \quad \Omega_{\mathrm{m}}=0.290 \pm 0.048
$$



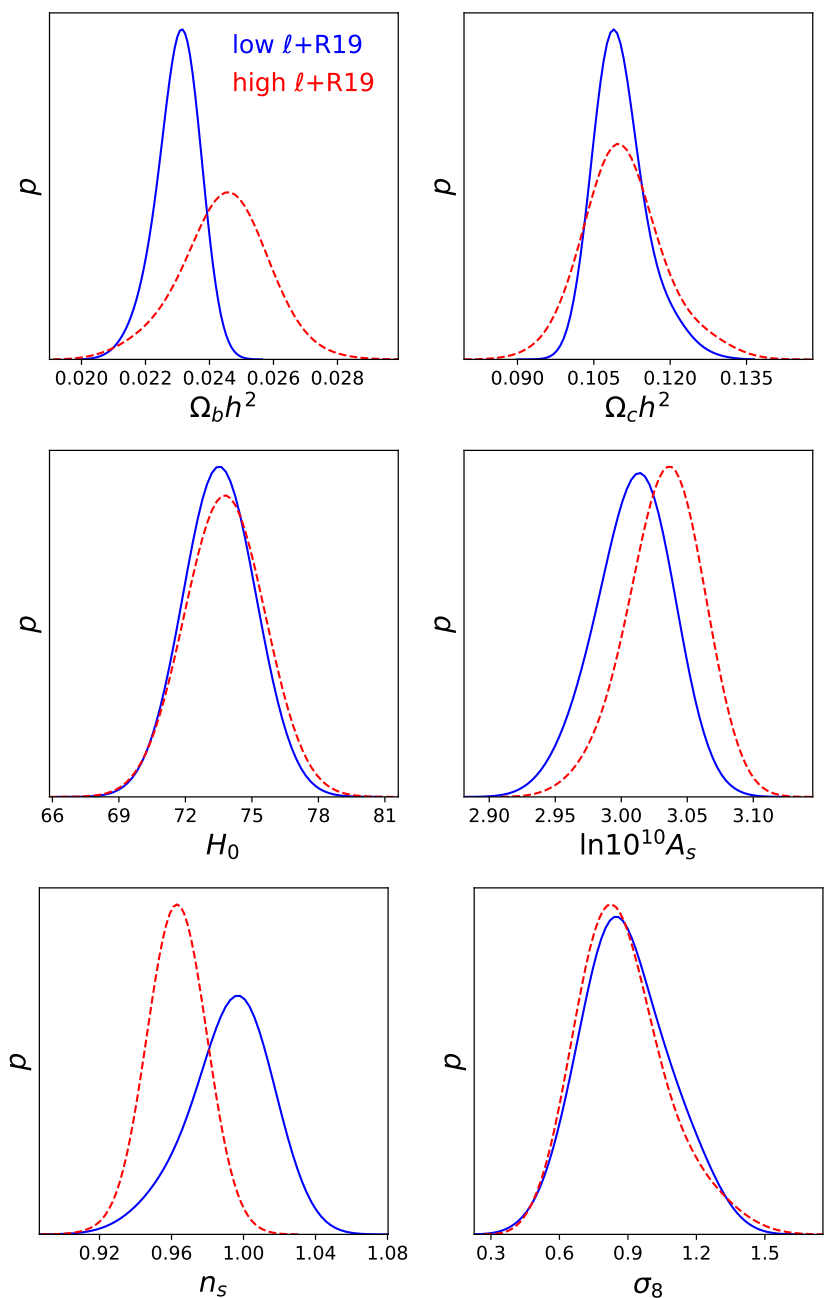

FIG. 3.- The marginalized likelihoods for various standard cosmological parameters in the GPT model as measured by low and high $\ell$ Planck temperature and low- $E$ anisotropies, with the R19 prior on $H_{0}$. The measurements from the two multipole regimes are found to be consistent.

from Pl18, and

$$
S_{8}=0.696 \pm 0.134, \quad \Omega_{\mathrm{m}}=0.281 \pm 0.053
$$

from DES. We also verified the consistency of the GPT parameters as measured by the individual Pl18 and DES datasets. We therefore conclude the consistency of the GPT cosmological inferences from these two datasets.

\section{RESULTS II: INTERNAL (IN)CONSISTENCIES IN PLANCK}

Here we explore whether the GPT scenario can explain the reported internal curious features of Pl18 when interpreted in the $\Lambda \mathrm{CDM}$ framework. These include the parameter shifts when separately inferred from highand low- $\ell$ regimes and the preference of the temperature power spectrum for stronger lensing than is required by $\Lambda C D M$. It is suggested that non-zero spatial curvature can resolve the latter, however, at the cost of increased $H_{0}$ and $\sigma_{8}$ tensions (Di Valentino et al. 2019).

\section{1. low-high $\ell$ tension}
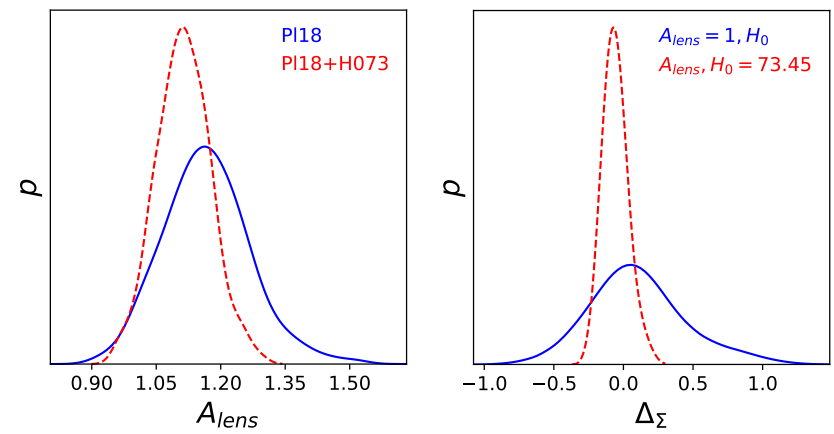

FIG. 4.- Left: Marginalized likelihoods of the lensing amplitude in the GPT framework, as measured by Pl18 (solid blue) and Pl18+H073 (dashed red). The $\Lambda$ CDM tension of $A_{\text {lens }}$ is reduced to less than a $\sigma$ in GPT. The Pl18+H073 curve shows that possible tight future $H_{0}$ constraints, even if far from the current $\Lambda \mathrm{CDM}$ inferences, will not reduce the $A_{\text {lens }}=1$ consistency in GPT.

Right: Marginalized likelihood for the derived lensing parameter $\Delta_{\Sigma}$ (see Equation 11) compared for two cases, one with $A_{\text {lens }}$ free and with a tight prior on $H_{0}$ through including H073 (red dashed curve) and the other with $A_{\text {lens }}$ set to unity and with the R19 prior on $H_{0}$ (solid blue curve). We see that the two likelihoods overlap, implying that the required $\Delta_{\Sigma}$ to get H073 does not conflict with the $\Delta_{\Sigma}$ needed to ensure $A_{\text {lens }}=1$.

We measure the different cosmic parameters in the GPT model separately with the low $(\ell \leq 800)$ and high $(800<\ell \leq 2500)$ multipoles of the Planck temperature anisotropies. Figure 3 compares the marginalized likelihoods for some of the standard parameters. In both cases, we also include the R19 (justified by its consistency with Pl18, see Section 4.1) and the Planck low-E, as otherwise the GPT parameters and the amplitude of primordial scalar perturbations would be unconstrained by the separate low and high $\ell$ data. The scalar index is the only parameter with a noticeable shift in its best-fit values. The measurements, however, are still consistent due to the notable overlap of the distribution ranges.

\section{2. lensing tension}

The lensing-specific dof $\Sigma$ in Eq. 5 is related to $\mu$ and $\gamma$ through

$$
\begin{aligned}
\Sigma & =-\frac{\mu}{2}(1+\gamma) \\
& \approx \Sigma^{\text {early }}+\Delta_{\Sigma} \frac{1+\tanh \left[\alpha\left(z_{t}-z\right)\right]}{2}
\end{aligned}
$$

where $\Delta_{\Sigma}=-\Delta_{\mu}-\Delta_{\gamma} / 2$ and the second line only includes linear contributions of $\Delta_{\mu}$ and $\Delta_{\gamma}$. The goal is to investigate whether modifications to $\Sigma$ in the GPT framework can resolve or reduce the tension in $A_{\text {lens. }}$. The left panel in Figure 4 illustrates the distribution of $A_{\text {lens }}$ in GPT as measured by Pl18, without and with a tight $H_{0}$ prior, labeled as $\mathrm{Pl} 18$ and $\mathrm{Pl} 18+\mathrm{H} 073$ respectively. We find that $A_{\text {lens }}$ is consistent with one in both cases. The futuristic prior imposed by H073 would tighten the bound and push the measurement toward lower amplitudes, without reducing the $A_{\text {lens }}=1$ consistency. We conclude that the GPT model would continue to explain the Pl18 data if the current high local Hubble value is measured with much higher accuracy.

It is also important to verify the consistency of the required lensing perturbations for the independent resolution of the lensing and Hubble tensions. We consider 

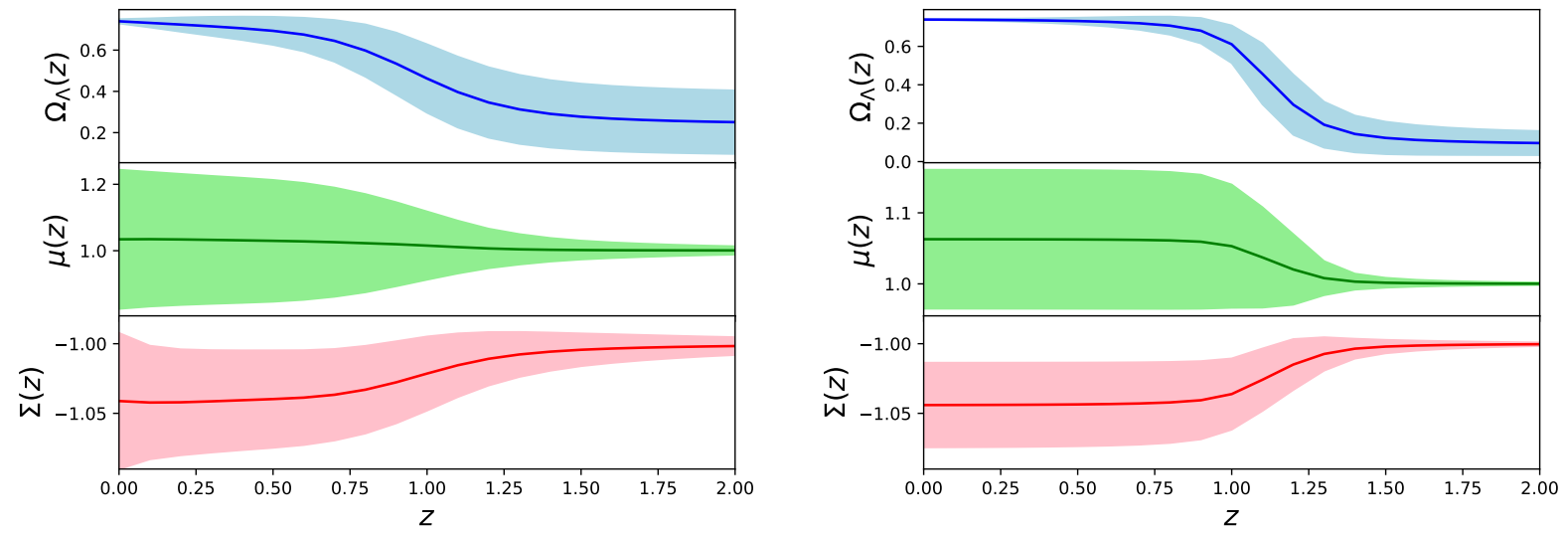

FIG. 5.- The $1 \sigma$ trajectories in redshift space traced by the various GPT parameters with the joint datasets Pl18+R19 (left) and Pl18+lensing+H073+DES (right). These transitions can relax the Planck external tensions and internal inconsistencies addressed in this work. The deviations in $\Omega_{\Lambda}(z)$ and $\Sigma(z)$ from their $\Lambda$ CDM values are mainly imposed by $H_{0}$ priors, most vivid in the right panel.

two observational scenarios, each with a tight prior on one of the two parameters $H_{0}$ and $A_{\text {lens }}$, and with the goal to measure the other parameter. The right panel of Figure 4 compares the likelihoods for the two cases, one with Pl18+H073 with $A_{\text {lens }}$ free (the dashed red curve) and one with Pl18 with $A_{\text {lens }}=1$ and $H_{0}$ free (the blue solid curve). The two likelihoods overlap, verifying that the two requirements in their extreme cases can be simultaneously satisfied with agreeing lensing corrections.

\section{CONCLUSION}

The analysis of the previous sections illustrates that the discussed Planck external $\left(H_{0}\right.$ and $\left.\sigma_{8}\right)$ tensions and internal (low-high $\ell$ and lensing amplitude) inconsistencies are relaxed in the GPT framework. The best-fit trajectories and their $1 \sigma$ confidence regions in the left panel of Figure 5 show the transitions in $\Omega_{\Lambda}(z), \mu(z)$ and $\gamma(z)$ required to relax the Hubble tension. We have set $A_{\text {lens }}=1$ as its consistency with R19 (and H073) was shown in Section 5.2. The best-fit trajectories of all three amplitudes show preference for time variations and smoothly go to their early-time values. However, the shaded $1 \sigma$ regions surrounding the best-fit trajectories show that $\mu$ is fully consistent with GR while $\Sigma$ and $\Omega_{\Lambda}$ noticeably deviate from their $\Lambda \mathrm{CDM}$ values.

It is interesting to see how tighter futuristic Hubble measurements would constrain the trajectories. This is shown in the right panel of figure 5, where we have used the full data combination of Pl18+lensing +H073+DES. The lensing consistency and Hubble constraint assumptions mainly impact the $\Lambda(z)$ and $\Sigma(z)$ trajectories. One expects from Equation 4 that $\mu(z)$ would be best constrained by measurements of matter distribution, characterized, e.g., by tight bounds on $\sigma_{8}$. However, these measurements are model-dependent and it is not feasible to directly assume a tight prior on $\sigma_{8}$ to investigate whether and how it would be compensated by a proper transition in $\mu(z)$. One could, instead, simulate observations (of, e.g., power spectrum of cosmic shear or galaxy clustering) for an assumed set of GPT parameters, and see how those would lead to inconsistencies in the parameter measurements if analyzed in the $\Lambda \mathrm{CDM}$ framework. We did not explore this here.

We therefore find a late-time gravitational transition, simultaneous in the dof's of the background equation and the perturbed Einstein equations, relaxes the main current external Planck tensions, as well as its internal inconsistencies. The framework of phase transition also opens the room to address the spatial anomaly as was investigated in Banihashemi et al. (2019). We thus view the GPT scenario as a rich mechanism to encompass various datasets and phenomenologically address their temporal and spatial tensions and anomalies. In this work our focus was on the three datasets of Pl18(+lensing), R19 and DES. In future analysis one should also include a broader set of data, such as BAO and high- $z$ supernovae. The next natural extension is to allow for the early phase of gravity to deviate from GR. A main concern would then be the many dof's which, if not dealt with properly, will inflate the parameter space and leave the parameters practically unmeasurable. This will be explored in future work.

\section{ACKNOWLEDGEMENT}

The numerical calculations of this work were carried out on the computing cluster of the Canadian Institute for Theoretical Astrophysics (CITA), University of Toronto.

\section{REFERENCES}

Abbott, T., et al. 2018, Phys. Rev. D, 98, 043526

Banihashemi, A., Khosravi, N., \& Shirazi, A. H. 2019, Phys. Rev. D, 99, 083509

- 2020, Phys. Rev. D, 101, 123521

Bloomfield, J. K., Flanagan, E. E., Park, M., \& Watson, S. 2013, JCAP, 08, 010
Braglia, M., Ballardini, M., Emond, W. T., Finelli, F.

Gumrukcuoglu, A. E., Koyama, K., \& Paoletti, D. 2020, Phys. Rev. D, 102, 023529

Di Valentino, E., Melchiorri, A., \& Mena, O. 2017, Phys. Rev. D, 96, 043503

Di Valentino, E., Melchiorri, A., Mena, O., \& Vagnozzi, S. 2020a,

Phys. Dark Univ., 30, 100666

-. 2020b, Phys. Rev. D, 101, 063502 
Di Valentino, E., Melchiorri, A., \& Silk, J. 2019, Nature Astron., 4, 196

Di Valentino, E., Mukherjee, A., \& Sen, A. A. 2020c

Freedman, W. L., et al. 2019

Gómez-Valent, A., Pettorino, V., \& Amendola, L. 2020, Phys. Rev. D, 101, 123513

Gubitosi, G., Piazza, F., \& Vernizzi, F. 2013, JCAP, 02, 032

Heymans, C., et al. 2020, arXiv e-prints, arXiv:2007.15632

Huang, C. D., et al. 2019

Keeley, R. E., Joudaki, S., Kaplinghat, M., \& Kirkby, D. 2019, JCAP, 12,035

Keeley, R. E., Shafieloo, A., Hazra, D. K., \& Souradeep, T. 2020, JCAP, 09, 055

Khetan, N., et al. 2020

Khosravi, N., Baghram, S., Afshordi, N., \& Altamirano, N. 2019, Phys. Rev. D, 99, 103526

Li, X., \& Shafieloo, A. 2019, Astrophys. J. Lett., 883, L3

Lucca, M., \& Hooper, D. C. 2020

Percival, W. J., et al. 2002, Mon. Not. Roy. Astron. Soc., 337, 1068
Pesce, D., et al. 2020, Astrophys. J. Lett., 891, L1

Planck Collaboration et al. 2020a, A\&A, 641, A6

-. 2020b, A\&A, 641, A7

Poulin, V., Smith, T. L., Karwal, T., \& Kamionkowski, M. 2019, Phys. Rev. Lett., 122, 221301

Raveri, M. 2020, Phys. Rev. D, 101, 083524

Riess, A. G., Casertano, S., Yuan, W., Macri, L. M., \& Scolnic, D. 2019, Astrophys. J., 876, 85

Riess, A. G., et al. 2016, Astrophys. J., 826, 56

-. 2018, Astrophys. J., 861, 126

Vattis, K., Koushiappas, S. M., \& Loeb, A. 2019, Phys. Rev. D, 99,121302

Wong, K. C., et al. 2020, Mon. Not. Roy. Astron. Soc., 498, 1420

Yan, S.-F., Zhang, P., Chen, J.-W., Zhang, X.-Z., Cai, Y.-F., \& Saridakis, E. N. 2020, Phys. Rev. D, 101, 121301

Yang, W., Mukherjee, A., Di Valentino, E., \& Pan, S. 2018a, Phys. Rev. D, 98, 123527

Yang, W., Pan, S., Di Valentino, E., Nunes, R. C., Vagnozzi, S. \& Mota, D. F. 2018b, JCAP, 09, 019

Zhao, G.-B., et al. 2017, Nature Astron., 1, 627 